likely to find RRESDO acceptable in retrospect $(\mathrm{OR}=5.3$, $95 \% \mathrm{CI}=1.2-27.5, \mathrm{p}<0.031) .88 .8 \%(143 / 161)$ premenopausal versus $95.2 \%(80 / 84)$ postmenopausal women who underwent RRSO respectively were satisfied with their decision. 9.4\%(15/ 160) premenopausal and $1.2 \%(1 / 81)$ postmenopausal women who underwent RRSO regretted their decision. HRT-uptake in breast-cancer (BC) unaffected premenopausal individuals was 74.1\% (80/108). ). HRT-use did not significantly affect satisfaction/regret levels but reduced symptoms of vaginal-dryness $(\mathrm{OR}=0.4,95 \% \mathrm{CI}=0.2-0.9, \mathrm{p}=0.025)$.

Conclusion Data show high RRESDO acceptability particularly in women concerned about sexual-dysfunction. Although RRSO satisfaction remains high, regret rates are much higher for premenopausal women than postmenopausal women. HRT use following premenopausal RRSO does not increase satisfaction and reduces vaginal dryness.

Disclosures Funding: Rosetrees Trust.

RM declares research funding from Barts Charity and Eve-Appeal outside this work, honorarium for advisory board membership from Astrazeneca/MSD, and from Israel National Institute for Health Policy Research, support from NHS Innovation Accelerator Fellowship. UM has a financial interest in Abcodia, Ltd., a company for academic and commercial development of biomarkers for screening and risk prediction. DGE declares honoraria for advisory board membership from Astrazeneca. The other authors declare no conflict of interest.

\section{SURGICAL DECISION MAKING IN PREMENOPAUSAL BRCA CARRIERS CONSIDERING RISK REDUCING EARLY- SALPINGECTOMY OR SALPINGO-OOPHORECTOMY: A QUALITATIVE STUDY}

${ }^{1}$ Faiza Gaba, ${ }^{2}$ Dalya Marks, ${ }^{3}$ Ertan Saridogan, ${ }^{4} W$ Glenn Mccluggage, ${ }^{5} \mathrm{Helen} H a n s o n$ ${ }^{6}$ Naveena Singh, ${ }^{2}$ Rosa Legood, ${ }^{7} \mathrm{D}$ Gareth Evans, ${ }^{3}$ Usha Menon, ${ }^{1}$ Ranijit Manchanda. ${ }^{1}$ Wolfson Institute of Preventive Medicine; Barts Health NHS Trust; Gynaecological Oncology; ${ }^{2}$ London School of Hygiene and Tropical Medicine; ${ }^{3}$ University College London Hospitals NHS Foundation Trust; ${ }^{4}$ Belfast Health and Social Care Trust; ${ }^{5}$ St George's University Hospitals NHS Foundation Trust; ${ }^{6}$ Barts Health NHS Trust; ${ }^{7}$ University of Manchester

\subsection{6/ijgc-2020-ESG0.161}

Introduction/Background Acceptance of the role of fallopian tubes in ovarian carcinogenesis and the detrimental sequelae of surgical menopause in pre-menopausal women following risk reducing salpingo-oophorectomy (RRSO), has resulted in risk reducing early salpingectomy with delayed oophorectomy (RRESDO) being proposed as an attractive alternative risk reducing strategy in women who decline/delay oophorectomy. We present the results of a qualitative study evaluating the decision making process amongst BRCA carriers considering prophylactic surgeries (RRSO/RRESDO) as part of the multicentre PROTECTOR trial (ISRCTN:25173360).

Methodology In depth semi-structured 1:1 interviews conducted using a pre-developed topic guide (development informed by literature review and expert consultation) until informational saturation reached. Wording and sequencing of questions were left open with probes used to elicit additional information. All interviews were audio recorded, transcribed verbatim, transcripts analysed using an inductive theoretical framework and data managed using NVIVO v12.

Results Informational saturation was reached following twenty four interviews. Seven interconnected themes integral to surgical decision making were identified: fertility, menopause, cancer risk reduction, surgical choices, surgical complications, sequence of ovarian and breast prophylactic surgeries, support, satisfaction. Women for whom maximising ovarian cancer (OC) risk reduction was relatively more important than early menopause/quality of life preferred RRSO, whereas those more concerned about detrimental impact of menopause chose RRESDO. Women preferred educational support groups to online support groups to help with decision-making. Women engage concurrently in both $\mathrm{OC}$ and breast cancer (BC) prevention decision-making and we identified a demand for combined $\mathrm{OC}$ and $\mathrm{BC}$ prevention-surgery. While preventative surgery reduced anxiety, interviewees wished to be routinely offered an 'optional' (not compulsory) consultation with a psychologist. Women managed in specialist familial cancer clinic (FCC) settings compared to non-specialist settings received better quality care, improved HRT access and were more satisfied.

Conclusion Medical, physical, psychological, social contextual factors influence timing of risk reducing surgeries. RRESDO offers women delaying/declining premenopausal oophorectomy, particularly those concerned about menopausal effects, a degree of ovarian cancer risk reduction whilst avoiding premature menopause. Care of high risk women should be centralised to centres with specialist familial gynaecological cancer risk management services to provide a better quality, streamlined, holistic multidisciplinary approach.

Disclosures Funding: Barts Charity and Rosetrees Trust.

RM declares research funding from Cancer Research UK and The Eve Appeal outside this work, an honorarium for advisory board membership from Astrazeneca/MSD and Israel National Institute for Health Policy Research. RM is supported by an NHS Innovation Accelerator (NIA) Fellowship. The other authors declare no conflict of interest.

\section{PAP SMEAR SCREENING AMONG FEMALE PATIENTS OF THE IBN ROCHD UNIVERSITY HOSPITAL CENTER: A CROSS SECTIONAL SURVEY}

${ }^{1}$ Asmàa Fouad, ${ }^{1}$ Nabila Youssouf, ${ }^{1}$ Fadwa Atfi, ${ }^{2}$ Amine Lamrissi, ${ }^{2}$ Karima Fichtali, ${ }^{2}$ Said Bouhya. 'Uhc Ibn Rochd; Med VI Center for Gyneacologic and Breast Cancer Treatment; ${ }^{2}$ Uhc Ibn Rochd; Gynecology and Obstetrics Department

\subsection{6/ijgc-2020-ESGO.162}

Introduction/Background Cervical cancer is preceded by a period of pre-invasive state, and is characterized histologically by a broad spectrum of events ranging from cellular atypia to different degrees of cervical intraepithelial neoplasia before progressing to invasive cancer. The association between certain oncogenic (high-risk) strains of HPV and cervical cancer is well established. The purpose of this study is to highlight through the findings - the importance of emphasizing accurate information about cervical cancer and the purpose of Pap smear for Moroccan women.

Methodology This cross-sectional study was carried out among 500 female patients who had a pap screening at the gynecology and obstetrics department at the UHC Ibn Rochd over a period of 2 years (2016 - 2017).

Results The average age of the patients having a pap smear screening is 39.5 years. The most affected age group is between 30 and 40 years old. $67 \%$ of the patients had started sexual activity before the age of 20 . A history of recurrent 